\title{
Agonistas del receptor de GLP-1
}

\author{
GLP-1 receptor agonists \\ Hugo César Arbañil-Huamán
}

\begin{abstract}
Resumen
Se describe la evolución y descubrimiento de una molécula derivada del proglucagón con efecto incretínico, en la búsqueda de una molécula resistente al rápido metabolismo ocasionado por la DPP-4, se encontró y desarrolló moléculas como el Exendin 4 y posteriormente las moléculas sintéticas, específicas para el receptor de GLP-1 y resistentes al metabolismo de DPP-4 y con efecto beneficioso cardiovascular y renal en los estudios de seguridad antes de su aprobación, en particular los de larga acción y parenterales, no así los de absorción oral.
\end{abstract}

Palabras clave: Diabetes Mellitus tipo 2, tratamiento, agonistas receptor GLP-1.

\section{Abstract}

The evolution and discovery of a molecule derived from the proglucagon with incretinic effect is described. In the search for a molecule resistant to the rapid metabolism caused by DPP-4, it was found molecules such as Exendin 4 and subsequently the synthetic ones, specific to the GLP-1 receptor and resistant to the metabolism of DPP-4. It was also found that they had cardiovascular and renal advantages in the safety studies before their were approved, in particular the longacting and parenteral ones but not those of oral absorption.

Keywords: Diabetes Mellitus type 2, treatment, GLP-1 receptor agonists.

\section{Introducción}

En la década de los 80 se logró identificar una hormona derivada del metabolismo de la molécula de Proglucagon, producida en el intestino delgado por las células $\mathrm{L}$, que se le denominó GLP-1 (Glucagon-like peptide type 1) con efecto incretínico similar al descrito para el GIP (gastric inhibitory polypeptide) $)^{(1,2)}$, esto es incremento de la secreción de insulina dependiente de la glucosa y disminución del glucagon. Dado que el tiempo de vida media de estas hormonas es muy corto debido al efecto de la enzima dipeptidil peptidasa tipo 4 (DPP4), que las metaboliza rápidamente, se trazó dos estrategias, una incrementando el tiempo de vida media de estas hormonas ${ }^{(3)}$; mediante inhibidores de esta enzima DPP-4 y la otra desarrollando agonistas de la GLP-1, que no sean metabolizadas por DPP-4. En ese proceso se demostró que estos medicamentos tenían un impacto importante en el control de la glucemia postprandial y que éste ocurría por el efecto de GLP-1 en retardar el vaciamiento gástrico, demostrándose al agregarse eritromicina que es un procinético, que disminuye el retardo en el vaciamiento gástrico ${ }^{(4,5)}$.

Por otro lado, se ha estudiado que los cardiomiocitos y las paredes arteriales tienen receptores para GLP-1 y que, experimentalmente en ratones, con infarto de miocardio cuando se les infundía GLP-1 se obtenía disminución del área infartada y mejoría de la función del ventrículo izquierdo ${ }^{(6)}$. Asimismo, experimentos realizados en ratones han mostrado efectos neuro-protectores que avizoran la posibilidad que puedan ser utilizados para el tratamiento de otras manifestaciones crónicas y complicaciones frecuentes en las personas con diabetes mellitus ${ }^{(7)}$.

\section{Cuerpo de Trabajo}

En el desarrollo de estos medicamentos, proviene de la observación que las personas que sufrían la mordedura de un reptil llamado "monstruo de Gila", les producía hipoglucemia. 
Por lo que se estudió su saliva, encontrándose una enzima que se descubrió tenía un $50 \%$ de semejanza con GLP-1, se le denominó exendina $4^{(8)}$; en el estudio se descubrió que se unía al receptor de GLP-1 y no era metabolizado como la molécula original de GLP-1, lo cual favorecía la acción hipoglucemiante. Con el conocimiento de esta molécula, se inicia la investigación de moléculas sintéticas que tengan acción sobre el receptor de GLP-1 y que sean resistentes a la acción de la DPP 4, la primera fue exenatida ${ }^{(9)}$. Después siguió lixisenatide, también una modificación de exendin $4^{(10)}$. Posteriormente, se empieza la síntesis de nuevas moléculas con características diferentes, sobre todo en el tiempo de vida media, lo cual ya les daba ventajas respecto a las iniciales derivadas de exendin 4 , en la tabla 1 vemos algunas diferencias.

\section{Farmacocinética}

Esta familia de medicamentos se administra por vía subcutánea y fueron cambiando desde 2 veces al día con la exenatida, que fue la primera en salir al mercado, hasta los a GLP1-R de aplicación diaria como lixisenaide y liraglutide; posteriormente, se desarrolló las formas de aplicación semanal, lo cual aumentó las ventajas de esta familia de drogas, es el caso de Semaglutide, Dulaglutide y Exenatide LAR. Mediante un favorecedor de la absorción oral, el sodio N-(8-[2hidroxilbonzoil] amino) caprilato o "SNAC", se incrementa la absorción de semaglutide en el estómago por incremento el pH local, lo cual lleva a aumento de solubilidad de la droga y la protege de la degradación proteolítica ${ }^{(11)}$. Esta droga fue aprobada el año pasado para ser usada en el control de la diabetes mellitus, una vez al día por vía oral, después de publicado el estudio Pioneer 6 que mostró no ser inferior respecto al placebo.

\section{Tratamiento - Guías}

En los últimos años ante la evidencia producida por diferentes estudios en el mundo, las guías han ido cambiando, la base del tratamiento continúa siendo la indicación en los cambios en el estilo de vida; la terapia con fármacos se debe iniciar sea con monoterapia o con una combinación, para evitar la inercia médica y obtener una memoria metabólica favorable

\begin{tabular}{|c|c|c|}
\hline Compa & $\begin{array}{c}\text { Tabla } 1 \\
\text { on entre AGLP1-R de cor }\end{array}$ & larga acción \\
\hline Parámetros & AGLP1-R deaccióncorta & AGLP1-R deacciónlarga \\
\hline \multirow[t]{4}{*}{ Tiempo de vida media } & Exenatide, lixisenatide & Albiglutide, Dulaglutide, \\
\hline & & Exenatide LAR, Liraglutide, \\
\hline & & Semaglutide \\
\hline & $2-5$ horas & 12 horas a varios dias \\
\hline Glucosa de ayunas & Reducción modesta & Reducción fuerte \\
\hline $\begin{array}{l}\text { Hiperglucemia } \\
\text { postprandial }\end{array}$ & Reducción fuerte & Reducción modesta \\
\hline Insulina de ayunas & Estimulación modesta & Estimulación fuerte \\
\hline Insulina postprandial & Reducción & Modesta estimulación \\
\hline Secreción de glucagon & Reducción & Reducción \\
\hline Tasa de vaciamiento & Enlentecimiento & No efecto \\
\hline gástrico & Reducción & Reducción \\
\hline Presión arterial & No efecto o pequeño & Aumento \\
\hline Frecuencia cardíaca & $\begin{array}{c}\text { aumento ( } 0 \text { a } 2 \text { latidos } x \\
\text { min) }\end{array}$ & $\begin{array}{c}\text { Moderado (2 a } 5 \text { latido } x \\
\text { min) }\end{array}$ \\
\hline Peso & Baja 1 a 5 kg & Baja 2 a 5 kg \\
\hline Náusea & $\begin{array}{c}20 \text { a } 50 \% \text {, va bajando en } \\
\text { semanas a meses }\end{array}$ & $\begin{array}{c}20 \text { a } 40 \% \text {, atenúa rápido en } \\
4 \text { semanas }\end{array}$ \\
\hline
\end{tabular}


en las que tienen una hemoglobina glucosilada $\geq 8 \%{ }^{(12)}$. El mayor y discutido cambio propuesto por la guía ADA/EASD, se refiere a la elección de la primera droga en los pacientes que ya tienen un evento cardiovascular o son portadores de enfermedad renal crónica, en los que se debe dilucidar la disyuntiva entre privilegiar el cuidado ante la hipoglucemia, o se desea bajar de peso o el problema económico es el mayor, para definir que medicinas se va a usar, para lo cual se debe tomar en cuenta las evidencias que proporcionan estudios como Leader, Sustain 6, Rewind, en los que los agonistas del receptor de GLP1 juegan un papel muy importante ${ }^{(13)}$.

\section{Seguridad cardiovascular y efectos secundarios}

El efecto secundario más frecuente con esta familia de medicamentos son las náuseas, que fluctúan entre 20 a $50 \%$, llamando la atención que las personas de extracción asiática tienen menos nauseas que las personas de raza blanca ${ }^{(14)}$. Los vómitos son otro efecto secundario presente, pero rápidamente va desapareciendo sobre todo con los de larga acción ${ }^{(15)}$. Las diarreas se presentan en el $10 \%$ de pacientes, la explicación fisiopatológica no es clara, habiéndose observado más en los de larga acción ${ }^{(16)}$. Se ha reportado algunos casos de pancreatitis durante los estudios clínicos con exenatide u otros a GLP1-R ${ }^{(17)}$; sin embargo, se debe tener en cuenta que la posibilidad de tener pancreatitis es el doble en personas con DM2 en comparación con las no diabéticas ${ }^{(18)}$, por ello parece ser prudente tener presente que ante personas con antecedentes de pancreatitis es preferible no usar esta familia de medicamentos, aunque hasta el momento la farmacovigilancia no muestre un aumento de esta patología durante su uso ${ }^{(19)}$, ni se haya demostrado que sea importante monitorear con enzimas pancreáticas para prevenir el evento ${ }^{(20)}$.

Dado que las células $\mathrm{C}$ de la tiroides, productoras de calcitonina, tienen receptores a GLP-1, y que en roedores, se ha visto que puede asociarse a estimulo, proliferación y aparición de cáncer medular de tiroides ${ }^{(21)}$, se recomienda no usar en personas con antecedentes de tumores NEM tipo 2; pese a que, en una década usando esta familia de medicamentos, no se ha reportado aumento en su incidencia ${ }^{(22.23)}$. Otros efectos de estos medicamentos son disminución del peso y baja de la presión arterial, pero fue inquietante encontrar un aumento de la frecuencia cardíaca, sobre todo en los AGLP1R de larga acción, lo cual hace sospechar un impacto en la salud cardiovascular, la explicación estaría en el bloqueo de acción del nervio vago que se ha observado experimentalmente ${ }^{(24)}$, esta interrogante se terminó de despejar con los resultados de los estudios de seguridad cardiovascular. Para el caso de exenatide de larga acción el resultado no fue inferior respecto a placebo ${ }^{(25)}$. El estudio Elixa, usando lixisenatide (también derivado también de la exendina 4) no mostró inferioridad respecto a placebo en pacientes con síndrome coronario agudo, es decir mostró seguridad cardiovascular ${ }^{(26)}$.

El primer estudio que mostró ventajas cardiovasculares de esta familia fue el estudio Leader con liraglutide, el cual demostró superioridad comparado al tratamiento estándar + placebo, disminuyendo el riesgo relativo en $13 \%$ con significado estadístico para el objetivo primario consistente en mortalidad cardiovascular, IMA no fatal y enfermedad vascular cerebral no fatal, (MACE); cuando se analiza cada evento MACE, en la mortalidad cardiovascular el riesgo relativo bajó significativamente en $22 \%{ }^{(27)}$. Otro hallazgo favorable en dicho estudio fue la disminución del avance de la enfermedad renal. En el estudio Sustain 6 con semaglutide se demostró superioridad sobre placebo, con un impacto mayor sobre la enfermedad vascular cerebral ${ }^{(28)}$. En el estudio Rewind con dulaglutide también se demostró superioridad, bajando el riesgo relativo en $12 \%$ con significado estadístico y teniendo en cuenta que comparando al resto de estudios con esta familia de drogas, la población estudiada era predominantemente de prevención primaria ${ }^{(29)}$. En el estudio de seguridad cardivascular PIONNER 6, la Semaglutide oral demostró seguridad cardiovascular, pero sin las ventajas de la misma droga administrada por vía parenteral ${ }^{(30)}$; esto se explicaría porque, al momento de hacer este estudio, el porcentaje de pacientes que ya usaban iSGLT2 (inhibidores de cotransportador de sodio glucosa tipo 2) fue mayor que en el Sustain 6, ocultándose el efecto favorable.

\section{Era COVID y AGLP1-R}

En medio de la pandemia ocasionada por el SARS COV-2, el conocimiento que el coronavirus que ocasionó el MERS (síndrome respiratorio del oriente medio) ingresa al organismo humano usando DPP-4, planteó la probabilidad que agentes como AGLP1-R serian protectores en parte. Indudablemente el uso de insulina es preferido, pero se reporta que el uso de AGLP1-R pueden interferir con la disposición de tratamientos con atazanavir ${ }^{(31)}$. Por lo demás parecen ser seguros en su uso en estas circunstancias. 
Referencias bibliográficas

1. Gutniak M, Ørskov C, Holst JJ, Åhren B, Efendic S. Antidiabetogenic effect of glucagon-like peptide-17-36 amide in normal subjects and patients with diabetes mellitus. N Engl J Med 1992;326:1316-1322.

2. Nauck MA, Bartels E, Ørskov C, Ebert R, Creutzfeldt W. Additive insulinotropic effects of exogenous synthetic human gastric inhibitory polypeptide and glucagon-like peptide-1-7-36 amide infused at nearphysiological insulinotropic hormone and glucose concentrations. J Clin Endocrinol Metab 1993;76: 912-917.

3. Hare KJ, Vilsbøll T, Asmar M, Deacon CF, Knop FK, Holst JJ. The glucagonostatic and insulinotropic effects of glucagon-like peptide 1 contribute equally to its glucose-lowering action. Diabetes 2010;59:1765-1770..

4. Meier JJ. Normalization of glucose concentrations and deceleration of gastric emptying after solid meals during intravenous glucagon-like peptide 1 in patients with type 2 diabetes. J. Clin. Endocrinol. Metab. 2003;88: 2719-2725.

5. Meier JJ, Kemmeries G, Holst JJ, Nauck MA. Erythromycin antagonises the deceleration of gastric emptying by glucagon-like peptide 1 (GLP-1) and unmasks its insulinotropic effect in healthy subjects. Diabetes 2005;54:2212-2218.

6. Nikolaidis LA. Effects of glucagon-like peptide-1 in patients with acute myocardial infarction and left ventricular dysfunction after successful reperfusion. Circulation 2004;109: 962-965.

7. McClean PL, Parthsarathy V, Faivre E, Holscher C. The diabetes drug liraglutide prevents degenerative processes in a mouse model of Alzheimer's disease. J Neurosci 2011;31:6587-6594 .

8. Eng J, Kleinman WA, Singh L, Singh G, Raufman JP. Isolation an characterisation of exendin-4, an exendin-3 analogue, from Heloderma suspectum venom: further evidence for an exendin receptor on dispersed acini from guinea pig pancreas. J. Biol. Chem. 1992;267:7402-7405.

9. Kendall DM. Effects of exenatide (exendin-4) on glycemic control over 30 weeks in patients with type 2 diabetes treated with metformin and a sulfonylurea. Diabetes Care 2005;28:1083-1091.

10. Werne U, Haschke G, Herling AW, Kramer W. Pharmacological profile of lixisenatide: a new GLP-1 receptor agonist for the treatment of type 2 diabetes. Regul Pept 2010;164:58-64.

11. Baekdal TA. Pharmacokinetics, safety and tolerability of oral semaglutide in subjects with hepatic impairment. J Clin Pharmacol. 2018t; 58(10): 1314-1323.

12. Guía ALAD sobre el diagnóstico control y tratamiento de la diabetes mellitus tipo 2 con medicina basada en evidencia, Edición 2019.

13. ADA. Pharmacologic aproaches to glycemic treatment: standard of medical care 2019. Diabetes Care 2019;42(Suppl. 1):S90-S102.

14. Kaku K, Rasmussen MF, Clauson P, Seino Y. Improved glycaemic control with minimal hypoglycaemia and no weight change with the once-daily human glucagon-like peptide-1 analogue liraglutide as add-on to sulphonylurea in Japanese patients with type 2 diabetes. Diabetes Obes Metab 2010;12:341-347.
15.Lovshin JA, Drucker DJ. Incretin-based therapies for type 2 diabetes mellitus. Nat Rev Endocrinol. 2009;5:262-269.

16.Drucker DJ. Exenatide once weekly versus twice daily for the treatment of type 2 diabetes: a randomised, open-label, noninferiority study. Lancet 2008; 372:1240-1250.

17.Elashoff M, Matveyenko AV, Gier B, Elashoff R, Butler PC. Pancreatitis, pancreatic, and thyroid cancer with glucagon-like peptid 1-based therapies. Gastroenterology 2011; 141: 150-156.

18. Noel RA, Braun D K, Patterson RE, Bloomgren GL. Increased risk of acute pancreatitis and biliary disease observed in patients with type 2 diabetes: a retrospective cohort study. Diabetes Care 2009;32:834-838 .

19. Garg R, Chen W, Pendergrass M. Acute pancreatitis in type 2 diabetes treated with exenatide or sitagliptin: a retrospective observational pharmacy claims analysis. Diabetes Care 2010;33:2349-2354.

20.Lankisch P. Hyperamylasaemia and/or hyperlipasaemia: incidence and underlying causes in hospitalized patients with nonpancreatic diseases. Scand J Gastroenterol 2009;44:237-241.

21. Hegedus C. Calcitonin levels in rodents and humans basal and stimulated. J Clin Endocrinol Metab 2011;96:853-860.

22. Knudsen B. Experimental use of liraglutide on C cells. Endocrinology 2010;151:1473-1486.

23. Hegedus L, Moses AC, Zdravkovic M, Le Thi, T, Daniels GH. GLP-1 and calcitonin concentration in humans: lack of evidence of calcitonin release from sequential screening in over 5000 subjects with type 2 diabetes or nondiabetic obese subjects treated with the human GLP-1 analog, liraglutide. J. Clin. Endocrinol. Metab. 2011;96:853-860.

24.Imeryuz N. Glucagon-like peptide-1 inhibits gastric emptying via vagal afferent-mediated central mechanisms. Am J Physiol 1997;273:G920G927.

25. Holman RR, Bethel MA, Mentz RJ, Thompson VP, Lokhnygina Y, Buse JB et al. On behalf of the EXSCEL Study Group. Effects of OnceWeekly Exenatide on Cardiovascular Outcomes in Type 2 Diabetes. N Engl J Med 2017;377:1228-1239.

26.Pfeffe MA. Lixisenatide in Patients with Type 2 Diabetes and Acute Coronary Syndrome, N Engl J Med 2015;373:2247-2257.

27. Steven P, Marso MD. Liraglutide and cardiovascular outcomes in type 2 diabetes mellitus. N Engl J Med 2016;375(4):311-322.

28. Steven P, Marso MD. Semaglutide and cardiovascular outcomes in type 2 diabetes mellitus. N Engl J Med 2016; 375:1834-1844.

29. Gerstein H. Dulaglutide and cardiovascular outcomes in type 2 diabetes mellitus (Rewind): a double - blind randomized placebo - control trial. Lancet 2019;394:121-130.

30.Husain M. Oral semaglutide on cardiovascular outcomes in patients with type 2 diabetes mellitus. N Eng J Med 2019;381:841-851.

31.Sanders JM, Monogue ML, Jodlowski TZ, Cutrell JB. Pharmacologic treatments for coronavirus disease 2019 (COVID-19): a review. JAMA 13 April 2020 [Epub ahead of print]. DOI: 10.1001/jama.2020.601943.

Contribución de autoría: Hugo César Arbañil-Huamán ha sido el autor del estudio, contribuyendo con concepción, búsqueda electrónica, revisión inicial, el diseño de estudio, redacción, y revisión final.

Conflicto de interés: El autor no tiene conflictos de interés con la publicación de este trabajo.

Financiamiento: Autofinanciado.

Citar como: Arbañil-Huamán HC. Agonistas del receptor del GLP-1. Diagnóstico (Lima). 2020;59(2):65-68.

DOI: https://doi.org/10.33734/diagnostico.v59i2.219

Correspondencia: Hugo César Arbañil Huamán Correo electrónico: harbanil@gmail.com 